•综述・

\title{
榕树-榕小蜂互惠合作系统中的非对称性博栾
}

\author{
杨丽媛 $1,2,3$ 王瑞武 $4^{*}$ \\ 1 (浙江农林大学风景园林与建筑学院, 杭州 311300) \\ 2 (浙江省园林植物种质创新与利用重点实验室, 浙江农林大学, 杭州 311300) \\ 3 (南方园林植物种质创新与利用国家林业和草原局重点实验室, 浙江农林大学, 杭州 311300) \\ 4 (西北工业大学生态环境学院, 西安 710129)
}

摘要: 榕树及其传粉榕小蜂是自然界中目前所知道的关系最为紧密的互利共生系统之一。随着研究的深入, 越来 越多的证据发现榕树一传粉榕小蜂之间互惠合作的过程中存在着复杂的竞争和对抗关系, 例如榕树与传粉榕小蜂 之间对公共资源的竞争、传粉欺骗与宿主对传粉者的惩罚、榕树与传粉小蜂之间的“军备竞赛”等。在相互竞争或 者对抗关系中, 双方表现出非对称性相互作用。其非对称性关系主要表现出如下3个特征: (1)收益不对称, 即榕树 (宿主)与传粉榕小蜂(共生体)之间在资源利用等方面的实力不对称; (2)榕树与传粉榕小蜂之间的信息不对称; (3)进 化速率不对称。这些非对称的相互作用可能导致种群的波动、榕树与传粉榕小蜂相互适应和进化策略的变化。因 此, 理解榕树与传粉榕小蜂之间的非对称交互作用有助于理解为什么合作和冲突在互利共生关系中经常能同时存 在, 也将有助于解释榕树一传粉榕小蜂种间相互关系和物种的多样性。

关键词: 协同进化; 传粉欺骗; 寄主惩罚; 进化速率; 军备竞赛; 非对称性关系

\section{Asymmetric interactions in fig-fig wasp mutualism}

Liyuan Yang ${ }^{1,2,3}$, Ruiwu Wang ${ }^{4 *}$

1 Department of Ornamental Horticulture, School of Landscape Architecture, Zhejiang Agriculture and Forestry University, Hangzhou 311300

2 Zhejiang Provincial Key Laboratory of Germplasm Innovation and Utilization for Garden Plants, Zhejiang Agriculture and Forestry University, Hangzhou 311300

3 Key Laboratory of National Forestry and Grassland Administration on Germplasm Innovation and Utilization for Southern Garden Plants, Zhejiang Agriculture and Forestry University, Hangzhou 311300

4 School of Ecology and Environment, Northwestern Polytechnical University, Xi'an 710129

\begin{abstract}
Fig and fig-pollinating wasps constitute one of the most well-known systems of mutualistic interactions between species. However, interspecific competition and antagonism is increasingly observed in this obligate mutualism system, including competition over common resources, pollination cheating and host sanction, and an evolutionary arms race between the host tree and its pollinators. In the competitive and antagonistic interactions between fig and fig-pollinating wasps, three main asymmetric relationships have been identified: (1) asymmetric payoffs, i.e., asymmetric power between figs (host) and their pollinators (symbionts); (2) asymmetric rates of evolution; and (3) asymmetric information between figs and their pollinators. The asymmetric relationships may affect population dynamics and the mutual adaptation and evolutionary strategies of each species, which helps explain why both cooperation and conflict are simultaneously observed within a specific mutualism, and why diversified strategies and species coexistence are found in nearly all mutualism systems.
\end{abstract}

Key words: coevolution; pollination cheating; host sanction; evolutionary rate; arms race; asymmetric interaction

互利共生或者互惠关系在自然界中非常普遍，涉及的物种遍布自然界中的所有门类，几乎地球

收稿日期: 2020-06-09; 接受日期: 2020-08-06

基金项目: 国家自然科学基金(31700328)、陕西省自然科学基金(2019JQ-369)和浙江农林大学学校科研发展基金(2019FR028)

* 通讯作者 Author for correspondence. E-mail: wangrw@nwpu.edu.cn 
上的每一个物种都处于一种甚至多种互利互惠关 系中(Bronstein, 2001)。植物与传粉昆虫形成的互 利互惠关系是一种典型且非常普遍的互利共生关 系。其中, 榕树(桑科榕属Ficus)及其传粉榕小蜂 是目前所知道的自然界中关系最为紧密的互利合 作关系之一(Weiblen, 2002; Yang et al, 2004; Machado et al, 2005)。在二者的互利共生关系中, 榕树为榕小蜂提供抚养后代的营养以及繁殖的场 所, 榕小蜂为榕树传粉, 帮助榕树完成繁殖 (Janzen, 1979; Weiblen, 2002; Cook \& West, 2005)。

榕树与其传粉榕小蜂之间的协同进化历史长 达几百万年(Chen et al, 2010; Cruaud et al, 2012), 在长期的协同进化过程中, 二者之间在保持互惠 合作的同时, 也存在着竞争等对抗关系。在榕树 及其传粉榕小蜂的相互作用关系中, 对雌花资源 的利用、分配以及榕小蜂的传粉行为是二者合作 的基础, 也是竞争的焦点。榕小蜂为榕树传粉, 榕树为榕小蜂提供营养和栖息地, 二者之间表现 出合作关系(图1a); 在榕小蜂不携带花粉的情况 下, 榕树会对榕小蜂进行惩罚, 二者之间形成对 抗关系(图1b)。在雌花资源充足的情况下, 榕树与 传粉榕小蜂呈现出互惠合作关系; 但是在雌花资 源被双方过度利用, 雌花资源不足的情况下, 二 者之间会针对雌花资源产生竞争(图1c)。

相比于对称性竞争关系, 自然界中存在大量 的非对称性竞争关系: 比如动物竞争中, 存在着 双方体型大小、力量强弱的差异, 资源的拥有者 和非拥有者之间的差异等。竞争关系中双方不对 称的地位会对竞争的结果产生影响(Smith, 1982; 张大勇, 2000)。榕树是雌花资源的提供者, 使得 榕树与榕小蜂之间对雌花资源的竞争形成非对称 性竞争关系。在雌花资源的利用、分配以及传粉 行为的博亦过程中, 二者之间呈现出实力不对称 和信息不对称。在对雌花资源的利用和分配中, 榕树掌握着雌花资源(Murray, 1985), 使得榕树在 与榕小蜂的种群密度依赖竞争中占据了优势地位 (Yu et al, 2004; Wang et al, 2011)。在传粉行为的 博弯过程中, 虽然在传粉榕小蜂不执行传粉行为 的情况下, 榕树个体内部的资源分配能够减少甚 至不提供营养给这些榕小蜂(Peng et al，2008; Jander \& Herre, 2010), 但是不传粉的投机者能够
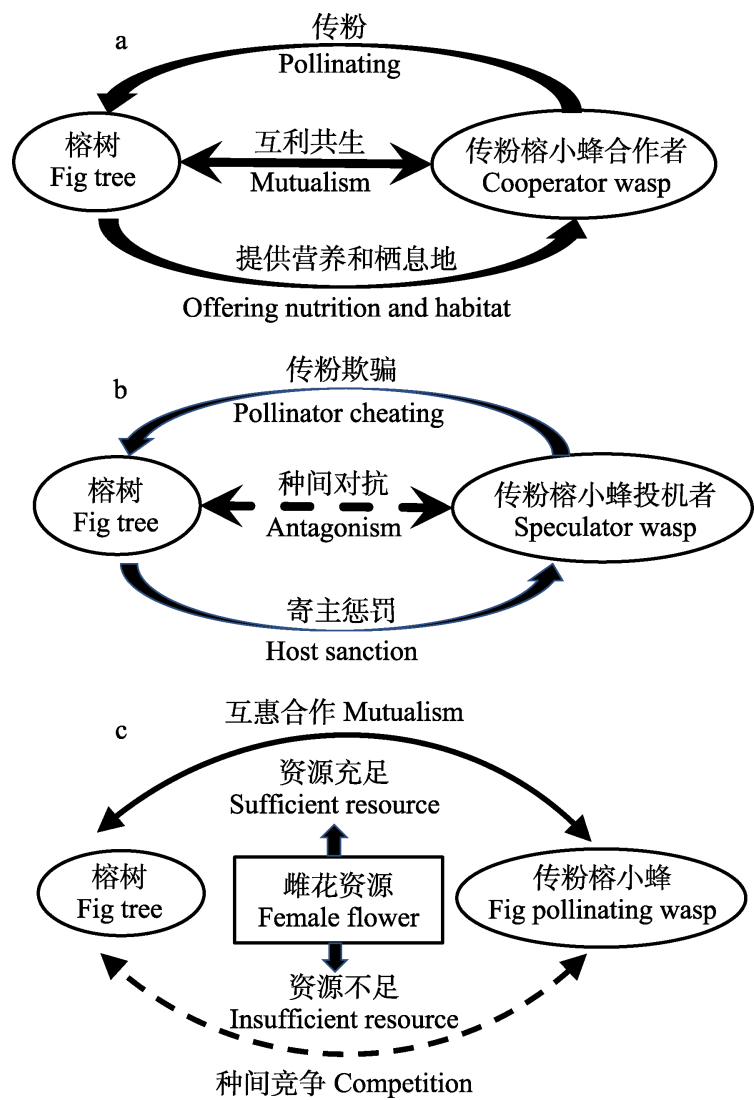

图1 榕树及其传粉榕小蜂之间的相互作用关系图。a, 榕 小蜂为榕树传粉, 榕树为榕小蜂提供营养和栖息地, 二 者之间表现为互利共生关系; $\mathbf{b}$, 传粉榕小蜂投机者不执 行传粉功能, 但是仍在利用榕树的资源, 榕树对投机者 进行寄主惩罚, 二者之间形成对抗关系。c, 在雌花资源 充足与否的两种情况下, 榕树与传粉榕小蜂表现出合作 关系或者竞争关系。本图根据Wang等(2011)整理。

Fig. 1 The interaction between figs and their fig pollinating wasps. a, the mutualism was based on figs offering nutrition and habitat to the wasp and the wasps pollinating the figs; $b$, pollination cheating and host sanction lead to antagonism relationship between fig and fig pollinating wasp; c, cooperation or competition between fig and fig pollinating wasp was dependent on the supply of common resource (according to Wang et al, 2011).

在传粉者个体的掩护下逃避榕树的惩罚。榕树并 不能够准确地判断特定的某个榕小蜂是否传粉, 其寄主惩罚的效力因此而打折扣，使得不传粉的 投机者个体能够在种群中保存下来(Jander \& Herre, 2016)。

在榕树与传粉榕小蜂协同进化过程中, 二者 世代更迭速度不同、进化速率不对称影响了它们 的进化历程以及相互对抗关系中的博弯策略。由 于榕小蜂的世代较短, 世代更迭速度和进化速率 较快(Frankham, 1996; Souto-Vilarós et al, 2019), 
短时间内积累了更多的基因突变(Herre et al, 1999), 为榕小蜂的行为多样性提供了物质基础。 对于传粉榕小蜂来说, 与榕树的博弯是单次的、 不重复的, 完成一次传粉和产卵, 亲本小蜂就结 束了使命和生命(Weiblen, 2002), 其策略的改变 只能通过代际之间的遗传突变来完成; 而对于榕 树来说，一棵榕树与传粉榕小蜂之间的博弯是多 次的, 每一季榕树的策略或形态特征都可以发生 变化, 其策略的变化通过表型可塑性来实现。由 于进化速率的不对称，榕树与传粉榕小蜂之间的 博弯策略演变的物质基础不同，策略多样性的产 生方式也不同。

榕树与传粉榕小蜂之间合作与竞争的关系随 着条件和环境的变化而转变(Wang et al, 2011, 2015b)。公共资源(雌花)是否充足，会影响资源分 配过程中榕树和小蜂之间的相互作用关系。榕树 和传粉榕小蜂之间的非对称性关系导致二者后代 适合度收益存在不确定性，促进二者博弯策略和 行为的多样性。在长期的协同进化过程中，榕树 和榕小蜂在竞争博弯过程中的行为多样性将可能 在基因水平上固定下来，促进遗传多样性及物种 多样性的形成。本文将着重探讨榕树与传粉榕小 蜂相互作用关系中的实力不对称、信息不对称以 及进化速率不对称，及其对榕树榕小蜂互利共生 关系的影响。

\section{种间竞争中的实力不对称}

即使是同一个体的基因组上也存在着不同基 因之间的利益冲突，互利共生系统内部的相互作 用关系也不是绝对和谐的(Herre et al, 1999)。榕树 与传粉榕小蜂之间潜在的利益冲突主要集中在繁 殖上对彼此的依赖。榕小蜂繁殖后代的营养全部 来自于榕果，榕树可以通过控制榕果的进蜂量来 控制榕小蜂的种群密度(Yu et al, 2004; Wang et al, 2011); 雌花资源分配会影响榕树和榕小蜂的繁 殖成功率, 对雌花的竞争更是形成了产卵器与花 柱长度之间的军备竞赛(Weiblen，2004); 榕小蜂 的传粉行为是榕树繁殖的保障, 榕树对于榕小蜂 传粉成功率 (诱骗小蜂为雌树传粉) (Weiblen, 2002; Cook \& Rasplus, 2003), 传粉的忠诚度(惩 罚欺骗者) (Guan et al, 2007)以及传粉效率(从主 动传粉中收益) (Kjellberg et al, 2001)方面, 都形
成了相应的对策。在这一系列的冲突中，榕树是 资源的提供者和控制者，在冲突中享有更高的 权利。

\section{1 种群密度依赖的种间竞争}

榕树与传粉榕小蜂的种群密度具有很大差异, 一株榕树一季可以结出成百上千个榕果，每个榕 果内可以养育几十到数千头小蜂。在榕树 - 传粉榕 小蜂之间的密度依赖的种间竞争中，榕树是优势 方(Yu et al, 2004; Wang et al, 2011)。榕树通过限 制进入榕果的小蜂数量来控制传粉榕小蜂对雌花 资源的利用，从而调节传粉榕小蜂的种群数量, 以达到调控种间竞争强度的目的。

隐头花序是榕属植物的特有特征。隐头花序 的结构限制了榕树传粉者的种类，使得只有专一 性的传粉榕小蜂才能够为榕树传粉。隐头花序的 结构也便于榕树对传粉者的控制, 尤其是对传粉 者种群密度的控制(Yu et al，2004; Wang et al, 2011)。在榕果接受期，在没有榕小蜂及时访问并 进入榕果(隐头花序)的情况下，榕果能够保持数 天的接受期状态，某些榕树物种如聚果榕 ( F. racemosa)、鸡嗉子榕(F. semicordata)的苞片口会张 大以便传粉榕小蜂进入。但是如果有多只蜂短时 间内进入榕果，苞片口会迅速闭合，进入榕果的 通道迅速封紧(Wang et al, 2009, 2015a; 张媛等, 2014)。同时，榕树还会通过改变挥发性物质的成 分来吸引或者排斥传粉榕小蜂。当很少或者没有 榕小蜂进入榕果时，榕树会释放出挥发物来吸引 传粉榕小蜂; 当有足够的榕小蜂进入榕果后，挥 发物的内容和含量会发生改变, 不再吸引甚至拒 绝传粉榕小蜂靠近(Proffit et al, 2008; Chen et al, 2009; Gu et al, 2012)。

传粉榕小蜂后代的性比率的改变也能起到间 接调控其种群密度的效果(Zhang et al, 2004)。传 粉榕小蜂是单双倍型昆虫, 亲本雌蜂通过控制卵 子是否受精来控制后代的性比。在进蜂量较低的 情况下，小蜂后代近亲交配的概率更高，小蜂倾 向于产生更加偏䧳的性比率(Herre，1985，1987), 雌性个体数量更多能够使得种群更容易扩大。进 蜂量影响榕小蜂近交水平进而影响性比率的调节 过程需要连续几个世代才能产生调节种群大小的 效果。在密度依赖的种间竞争过程中, 榕树通过 控制进蜂量直接调控小蜂的种群数量; 而传粉榕 
小蜂仅能通过调控后代性比率来间接调控种群 大小。

\section{2 雌花资源竞争中的军备竞赛}

榕树与传粉榕小蜂之间对雌花的竞争表现出 对抗性和浪费性(Murray, 1985), 其中传粉榕小蜂 因军备竞赛而导致的产卵器长度浪费性增长, 在 雌雄同株榕树的传粉榕小蜂中是能够被观察和检 测到的(Weiblen, 2004)。

榕树的隐头花序是传粉榕小蜂抚育后代的唯 一场所(Janzen, 1979; Cook \& West, 2005)。传粉榕 小蜂将产卵器通过榕树的花柱插入雌花的子房, 小蜂的卵在雌花的子房内发育成长(Janzen, 1979; Weiblen, 2002)。只有当产卵器长度比花柱长的情 况下, 小蜂的产卵器才能到达子房, 进而成功产 卵。榕树的䧳花不能同时既抚育小蜂又产生种子, 所以榕树和小蜂在雌花的利用上形成资源竞争 (Weiblen, 2002; Wang et al, 2015a)。在雌雄异株的 榕树中, 雌树的雌花全部产生种子, 不养育小蜂, 而雄树的雌花全部养育小蜂，不产生种子，榕树 和传粉小蜂对榕树雌花利用不存在竞争。但是在 雌雄同株榕树中，小蜂和种子在同一个隐头花序 内发育，榕树和传粉小蜂对榕树雌花利用中存在 竞争(表1)。相比于雌雄异株榕树的传粉小蜂, 雌 雄同株榕树的传粉榕小蜂经历了花柱长度和产卵 器长度之间的军备竞赛, 因而进化出过长的产卵 器。Weiblen (2004) 曾经测量了 43种传粉榕小蜂, 发现雌雄异株榕树的传粉小蜂的产卵器都比较短
(28种), 而 $80 \%$ (12种)的雌雄同株榕树的传粉小 蜂的产卵器都比较长(表2)。

延长花柱长度与延长产卵器长度所付出的代 价差异巨大。对于榕树来说，对花柱生长进行的 营养投资只占繁育投资中很小的份额，但是对于 传粉榕小蜂来说, 过长的产卵器给飞行带来了巨 大的负担。Jander等(2016)发现，榕小蜂的体型大 小对榕小蜂能否顺利到达榕果的影响很大。对于 传粉榕小蜂来说，资源过度投入到产卵器生长上, 显然是一个巨大的负担。在竞争过程中，传粉榕 小蜂进化出更长的产卵器以便在更多的雌花中产 卵(Ganeshaiah et al, 1995; Weiblen, 2004; Ghana et al，2017); 而榕树通过增长花柱长度限制传粉榕 小蜂产卵，保证了种子对雌花资源的利用。

在种群/物种水平上, 榕树与其传粉榕小蜂之 间在花柱和产卵器长度上存在军备竞赛; 在不同 物种的水平上，花柱和产卵器长度之间存在着精 确的匹配关系(Weiblen, 2004)。花柱和产卵器长 度之间精确的共适应, 可能恰恰是在激烈的军备 竞赛之下权衡的结果。

\section{3 主动传粉行为给榕树带来的适合度收益}

榕小蜂是榕树忠诚而高效的传粉者。传粉榕 小蜂形态、行为的特化使其能够高效特异地将花 粉传给寄主榕树。很多传粉榕小蜂物种具有花粉 笚、花粉梳等结构，具有主动收集和散播花粉的 行为; 而另一部分传粉榕小蜂物种则通过身体粘 黏携带花粉，完成散粉(Cook \& Lopez-Vaamonde,

表1 榕树隐头花序和小花的性别功能分配

Table 1 Sex function allocation of fig flower and syconium

\begin{tabular}{|c|c|c|c|c|c|}
\hline & \multirow{2}{*}{$\begin{array}{l}\text { 雌雄同株 } \\
\text { Monoecy }\end{array}$} & \multicolumn{3}{|c|}{ 雌雄异株 Dioecy } & \multirow{2}{*}{$\begin{array}{l}\text { 性别功能 } \\
\text { Sexual function }\end{array}$} \\
\hline & & 雄树 & Male tree & 雌树 Female tree & \\
\hline 雄花发育产物 Product from male flower & 花粉 Pollen & 花粉 & Pollen & - & 雄性功能 Male function \\
\hline \multirow[t]{2}{*}{ 雌花发育产物 Product from female flower } & 小蜂 Wasp & 小蜂 & Wasp & - & \\
\hline & 种子 Seed & - & & 种子 Seed & 雌性功能 Female function \\
\hline
\end{tabular}

表2 不同的寄主榕树性系统与其传粉榕小蜂产卵器长度的对应关系

Table 2 Number of pollinating wasp species with different ovipositor length in different fig sexual systems

\begin{tabular}{llll} 
寄主榕树性系统 & $\begin{array}{l}\text { 产卵器长度大于腹部的传粉小蜂种数 } \\
\text { Ovipositor is longer than the thorax }\end{array}$ & $\begin{array}{l}\text { 产卵器长度短于腹部的传粉小蜂种数 } \\
\text { Ovipositor is shorter than the thorax }\end{array}$ & $\begin{array}{l}\text { 总计 } \\
\text { Total }\end{array}$ \\
\hline 雌雄同株 Monoecy & 12 & 3 & 15 \\
倠雄异株 Dioecy & 0 & 28 & 28 \\
总计 Total & 12 & 31 & 43 \\
\hline
\end{tabular}

数据来自Weiblen (2004) Data were from Weiblen (2004) 
2001; Cook \& Rasplus, 2003)。

传粉榕小蜂的主动传粉行为给榕树带来的最 直接的收益就是节约花粉。针对 88 种传粉榕小蜂 及其寄主榕树的调查发现(Kjellberg et al, 2001), 在传粉者主动传粉的情况下, 榕树的花粉(花药) 数量较少, 其花药胚珠比(anther: ovule)小于 0.16 , 而不具有主动传粉者的榕树, 其花药胚珠比 (anther : ovule)在 0.21 以上(Kjellberg et al, 2001; Cook \& Rasplus, 2003)。

对于传粉榕小蜂来说, 主动传粉行为的收益 并不很明确(Kjellberg et al, 2001; Jousselin et al, 2003)。缺少花粉会导致传粉榕小蜂的后代数量和 质量下降(Jousselin et al, 2003; Wang et al, 2014b; Jander et al, 2016; Jander \& Herre, 2016); 另一方 面, 在物种水平上, 传粉榕小蜂彻底放弃主动传 粉行为似乎并没有太大的损失, 在被动传粉的榕 小蜂物种中, 花粉缺失似乎对榕小蜂后代的发育 影响不大(Peng et al, 2008; 徐睿等, 2016), 传粉 小蜂并不会因为不携带花粉而受到寄主榕树的惩 罚, 被动传粉的榕小蜂也可以在未授粉的雌花中 发育(Kjellberg et al, 2001; Jousselin et al, 2003)。

\section{4 榕树通过寄主惩罚控制欺骗者的出现}

主动传粉能够给榕树带来收益, 同时榕树需 要通过额外的手段来阻止不传粉的欺骗者的出 现。榕树可以通过 3 种方式来惩罚不传粉的榕小 蜂: (1)落果, 直接将全部小蜂后代杀死(Guan et al, 2007; Jander et al, 2012; Wang et al, 2014a; Zhang XW, 2019); (2)减少小蜂后代的数量 (Jander \& Herre, 2010; Jander et al, 2012); (3)降低小蜂后代 的质量(Jander et al, 2016)。寄主惩罚的方式可能 是有效的, 针对巴拿马 4 种传粉榕小蜂的调查发 现, 寄主惩罚的强度越强, 种群中出现不携带花 粉的个体(欺骗者)的概率就越低(Jander \& Herre, 2010)。并且榕树与传粉榕小蜂之间互利共生关系 的紧密程度会影响榕树对小蜂的惩罚强度：关系 越紧密, 惩罚强度越弱(Wang et al, 2015a)。榕树 所实施的寄主惩罚效应, 有效减少了欺骗者小蜂 的数量。在种群水平上, 寄主惩罚效应是维持榕 树-传粉榕小蜂互利共生关系的有力手段。

\section{信息不对称}

在榕树与传粉榕小蜂的竞争博弯过程中, 由
于彼此的信息不对称而发生了诸多欺骗事件，使 得榕树与传粉榕小蜂之间在个体水平上的博亦策 略变得更加丰富多样。

\section{1 雌树对传粉者的诱骗}

榕小蜂为榕树传粉是榕树-传粉榕小蜂之间 互惠合作的基础，但在雌雄异株榕树中，雄树抚 育小蜂，雌树生产种子，进入雌树榕果的小蜂不 能产生后代, 雌树的榕果对于传粉榕小蜂来说是 一个死亡陷阱(Weiblen，2002; Cook \& Rasplus, 2003)。所以选择压力使得传粉榕小蜂倾向于分辨 雌树和雄树, 找到与出生时一样的树(雄树)来完 成繁育后代。有证据表明雌雄异株榕树的传粉榕 小蜂对寄主榕树的识别能力更强, 识别错误率更 低(Yang et al, 2015)。然而, 雌树通过模仿雄树的 挥发物, 仍然能够成功吸引到传粉小蜂(Soler et al, 2012; Hossaert-McKey et al, 2016), 传粉榕小 蜂仍然不能避免进入雌树的榕果, 为榕树传粉却 不能完成自己的适合度 (Hossaert-McKey et al, 2010; Gu et al, 2012; Soler et al, 2012); 但是小蜂 保证了榕树种群的繁衍, 也就是保证了小蜂繁殖 后代所需的栖息地和营养来源。

\section{2 榕小蜂的传粉欺骗}

传粉榕小蜂在为榕树传粉的过程中并不总是 忠诚的, 不传粉的欺骗者以一定比例在种群中存 在(Jander \& Herre, 2010), 甚至存在传粉榕小蜂 进化成为欺骗者, 即稳定的不传粉的物种的现象 (Galil \& Eisikowitch, 1968; Compton et al, 1991; Peng et al, 2008; Zhang T et al, 2019)。对于传粉榕 小蜂来说, 逃避传粉是有益处的, 因为携带花粉 飞行需要耗费体力, 只有体型较大的小蜂才能成 功到达目的地(接受期榕果), 然而由于榕果苞片 口的特殊结构, 阻止了体型过大的小蜂进入榕果, 使得无论是过大或者过小的传粉者个体都不能成 功到达并进入榕果。在对高榕(Ficus altissima)的 调查发现, 到达榕果的传粉榕小蜂 Eupristina. altissima 明显比欺骗者 Eupristina sp. 的体型大, 而进入榕果的传粉榕小蜂却明显比欺骗者的体型 小(Xu et al, 2016)。并且, 欺骗者榕小蜂似乎并没 有因为不携带花粉而受到惩罚，其后代的适合度 并没有比传粉者低(Peng et al, 2008)。

不携带花粉的欺骗者能够在榕树-传粉榕小 蜂相互作用关系的博亦中找到有利的机会。西双 
版纳地区的小叶榕(Ficus microcarpa)的传粉欺骗 者似乎利用了传粉者种群密度季节性变动的特点, 在传粉者数量较少的季节大量利用榕树的闲置资 源来完成繁殖(Zhang $\mathrm{T}$ et al, 2019)。欺骗者似乎 在互惠关系更紧密的榕树-传粉榕小蜂系统中更 容易出现。例如在巴拿马地区，主动传粉的榕小 蜂种群内存在欺骗者, 而被动传粉的榕小蜂种群 内反而没有欺骗者(Jander \& Herre, 2010)。目前发 现的 3 种欺骗者传粉小蜂, 在高榕和小叶榕中的 进蜂量都在1-2头/果(Ficus sycomorus的进蜂量无 数据), 进蜂量较小被认为是榕树与传粉榕小蜂 互惠关系更紧密的特征。这暗示了榕树与榕小蜂 在相互依赖程度更高时，彼此之间的博弯更容易 发生多样化的策略。

\section{3 寄主惩罚的局限性}

虽然榕树对传粉欺骗行为有一定的惩罚措施, 但是寄主惩罚的精确性却很差：当携带花粉的小 蜂(合作者)和不携带花粉的小蜂(欺骗者)进入同 一个榕果内产卵的时候，榕树不能够进行区分， 惩罚措施只能实施在整个榕果上(Jander et al, 2016)，这使得不携带花粉的个体能够在合作者 的掩护下繁育后代，完成适合度收益。在这种信 息不对称的情况下，欺骗者得以在种群中以一定 的比例存在。在单果进蜂量更大的物种中, 投机 者更容易得到传粉者的掩护，这可能解释了为什 么单果进蜂量大的榕树对投机者的惩罚强度更强 (Wang et al, 2015a)。

雌雄异株榕树的雌树和雄树的性别功能不同, 雄树主要承担养育传粉者、散播花粉的功能(表 1)。对于雄树来说，进入榕果的小蜂是否携带花 粉并无影响; 影响雄树适合度的是子代小蜂离开 榕果时是否会收集和携带花粉。有调查发现，同 一物种的雄树对不携带花粉的小蜂要比雌树宽容 得多(Zhang T et al, 2019)。这就给不携带花粉的 小蜂提供了在种群中保留下来的机会。

\section{3 榕树与传粉榕小蜂的进化速率不对称}

榕树与其传粉榕小蜂之间有着紧密的互利共 生关系和严格的协同进化关系(Cruaud et al, 2012), 可能促进进化速率的加快。针对 Pseudomyrmex 属蚂蚁的比较基因组研究发现, 在 亲缘关系较近的不同种蚂蚁之间，有共生习性的
蚂蚁比没有共生性的蚂蚁的进化速率要高(Rubin \& Moreau, 2016); 而榕属的物种多样性也明显比 桑科的其他属高, 桑科有 40 多个属, 共 1,000 多种, 其中榕属就有750多种(Moe et al, 2012)。“红皇后 假说” 非常形象地解释了互利共生关系加快进化 速率的现象。红皇后假说来源于《爱丽丝梦游仙 境》中红皇后对爱丽丝说的名言“你只有拼命奔跑, 才能够原地不动”。在持续的选择压力下, 互利共 生的双方会形成进化上的军备竞赛，导致双方都 尽可能快地进化(Herre et al, 1999; Thompson, 2013)。榕树与传粉榕小蜂之间紧密的互利共生关 系促进了二者的协同进化，甚至协同成种，进而 加速了二者的成种速率。

在榕树与传粉榕小蜂的协同进化过程中，两 者进化速率差异很大。榕树为木本植物, 包括高 大乔木和灌木以及藤本植物，寿命多在十几甚至 上百年(Berg et al, 2005), 然而传粉榕小蜂的寿命 通常仅1-2个月(Weiblen, 2002)。榕树与传粉榕小 蜂的世代周期长度差别很大：传粉榕小蜂一年可 以经历几个世代, 然而榕树的种子长成大树, 再 到开花结果则需要数年的时间。世代时间影响进 化速率(Sharp \& Li, 1989), 榕小蜂的基因突变率 也比榕树更高(Frankham, 1996; Cook \& Rasplus, 2003), 加之严重的近交因素等使榕小蜂的物种 分化往往快于榕树(Herre et al, 2008; Chen et al, 2010), 这就导致了榕小蜂的遗传多样性比榕树 高(Souto-Vilarós et al, 2019)。例如，频繁发现的 传粉榕小蜂隐种事件暗示了传粉榕小蜂的物种数 可能要比榕树更多(Molbo et al, 2003; Haine et al, 2006; Huang et al, 2018) 。

榕树与榕小蜂之间进化速率不对称使得二者 在博弯过程中使用的策略不同, 榕小蜂的行为多 样性以遗传突变为基础, 而榕树则通过表型可塑 性来产生策略的多样性。例如榕小蜂的传粉欺骗 行为在种群中固定下来可以通过遗传突变来解释, 而榕树则通过营养资源重新分配来完成对传粉榕 小蜂的惩罚(Jander \& Herre，2016)。Jander和 Steidinger (2017)通过模型模拟分析发现，巴拿马 地区的 4 种传粉榕小蜂种群中稳定存在的欺骗者 小蜂可能就是突变产生的。更进一步, 欺骗行为 的固定甚至可以促进物种的分化，已经分化成种 的欺骗者传粉小蜂物种也被证实是由传粉榕小蜂 
表3 榕树-榕小蜂在传粉行为中的非对称关系

Table 3 The asymmetric interaction between fig and fig pollinating wasp during the pollination conflict

\begin{tabular}{|c|c|c|c|c|}
\hline $\begin{array}{l}\text { 博弯策略 } \\
\text { Strategy }\end{array}$ & $\begin{array}{l}\text { 受益方 } \\
\text { Winner }\end{array}$ & $\begin{array}{l}\text { 博弯结果 } \\
\text { Result }\end{array}$ & $\begin{array}{l}\text { 非对称关系 } \\
\text { Asymmetric interaction }\end{array}$ & $\begin{array}{l}\text { 代表性文献 } \\
\text { Typical reference }\end{array}$ \\
\hline $\begin{array}{l}\text { 主动传粉 } \\
\text { Active pollinating }\end{array}$ & 榕树 Figs & $\begin{array}{l}\text { 榕树节约花粉 } \\
\text { Figs produce less pollens }\end{array}$ & $\begin{array}{l}\text { 实力不对称 } \\
\text { Asymmetric power }\end{array}$ & Kjellberg et al, 2001 \\
\hline $\begin{array}{l}\text { 传粉欺骗 } \\
\text { Pollinator cheating }\end{array}$ & 小蜂 Wasps & $\begin{array}{l}\text { 小蜂减少传粉负担 } \\
\text { Wasp takes less burden for carrying pollens }\end{array}$ & $\begin{array}{l}\text { 信息不对称 } \\
\text { Asymmetric information }\end{array}$ & Peng et al, 2008 \\
\hline $\begin{array}{l}\text { 寄主惩罚 } \\
\text { Host sanction }\end{array}$ & 榕树 Figs & $\begin{array}{l}\text { 防止欺骗者的出现 } \\
\text { Reducing the cheating wasps }\end{array}$ & $\begin{array}{l}\text { 实力不对称 } \\
\text { Asymmetric power }\end{array}$ & Jander \& Herre, 2010 \\
\hline $\begin{array}{l}\text { 传粉投机者 } \\
\text { Speculator }\end{array}$ & 小蜂 Wasps & $\begin{array}{l}\text { 小蜂逃避寄主惩罚 } \\
\text { The wasps avoid the host sanction }\end{array}$ & $\begin{array}{l}\text { 信息不对称 } \\
\text { Asymmetric information }\end{array}$ & Jander et al, 2012 \\
\hline $\begin{array}{l}\text { 雌树诱骗 } \\
\text { Female tree attracts wasp } \\
\text { by mimicking male tree }\end{array}$ & 榕树 Figs & $\begin{array}{l}\text { 小蜂失去繁殖机会 } \\
\text { Wasps fail to reproduce }\end{array}$ & $\begin{array}{l}\text { 实力不对称 } \\
\text { Asymmetric power }\end{array}$ & Cook \& Rasplus, 2003 \\
\hline $\begin{array}{l}\text { 基因突变产生欺骗者 } \\
\text { Mutation causes cheaters }\end{array}$ & 小蜂 Wasps & $\begin{array}{l}\text { 维持种群内投机者比例 } \\
\text { Stable proportion of cheaters in population }\end{array}$ & $\begin{array}{l}\text { 进化速率不对称 } \\
\text { Asymmetric evolution rates }\end{array}$ & $\begin{array}{l}\text { Jander \& Steidinger, } \\
2017\end{array}$ \\
\hline
\end{tabular}

经物种复制分化(speciation duplication)而来(Yang et al, 2015; Zhang T et al, 2019)。榕树对于欺骗者 的惩罚通过停止或减少对欺骗者后代的营养投入 来实现(Jander \& Herre, 2016)。在整个榕果没有进 入携带花粉小蜂(只有欺骗者进入)的情况下, 榕 果内没有种子形成, 榕树会放弃整个榕果(落果), 榕树的营养资源将重新分配给有花粉的(进入传 粉者的)榕果, 以保证榕树的种子产量(Guan et al, 2007; Zhang T et al, 2019)。对于同时有欺骗者和 传粉者进入的榕果, 榕果的种子较少, 榕树对榕 果投入资源量也减少, 导致传粉榕小蜂后代数量 和质量降低(Jander \& Herre, 2010; Jander et al, 2012)。榕树甚至能够精确地判断虫瘘的性别, 分 配给雌性幼虫比雄性幼虫更多的营养( Li et al, 2016)。

榕树花柱长度与传粉榕小蜂产卵器长度之间 的博弯也受到进化速率不对称的影响。对聚果榕 的研究发现, 随着纬度的变化, 花柱长度以及传 粉榕小蜂的产卵器长度也发生相应的变化(王瑞 武等, 未发表数据)。其中花柱长度的变化更可能 是由于环境作用导致的表型可塑性变化, 而榕小 蜂的产卵器长度的变异更可能是来源于遗传多 样性。

\section{4 总结}

在榕树与传粉榕小蜂之间长期的协同进化历 史中, 除了合作互惠关系之外也存在对资源的竞 争、对抗关系。榕树-传粉榕小蜂之间紧密的互惠 合作关系和多样的博亦策略, 使得榕树-传粉榕
小蜂系统成为研究竞争、对抗关系的最佳模型。 由于榕树与传粉榕小蜂之间的实力不对称和信息 不对称，使得二者在资源竞争、传粉行为冲突中 表现出丰富多样的策略(表3)。由于进化速率不对 称, 榕树与传粉榕小蜂博弯策略产生的来源和物 质基础不同，榕树通过表型可塑性产生的形态变 异与传粉榕小蜂由于遗传多样性而产生的行为多 样性之间发生博弯。在二者的竞争关系中, 在不 同的博亦策略下，榕树与榕小蜂的适合度收益也 不同。在榕树与传粉榕小蜂的竞争博弯中, 公共 资源(雌花)在榕树种子发育和小蜂后代抚育的分 配中存在不确定性，导致适合度收益存在不确定 性。榕树与榕小蜂丰富多样的形态特征和行为策 略在基因水平固定下来, 促进了种群遗传多样性 的发生，甚至形成物种分化，促进了物种多样性 的形成。

\section{参考文献}

Berg CC, Corner EJ, Nooteboom HP (2005) Flora Malesiana (Moraceae-Ficus). National Herbarium Nederland, Leiden.

Bronstein JL (2001) The exploitation of mutualisms. Ecology Letters, 4, 277-287.

Chen C, Song QS, Proffit M, Bessière JM, Li ZB, Hossaert-McKey M (2009) Private channel: A single unusual compound assures specific pollinator attraction in Ficus semicordata. Functional Ecology, 23, 941-950.

Chen Y, Li HQ, Liu M, Chen XY (2010) Species-specificity and coevolution of figs and their pollinating wasps. Biodiversity Science, 18, 110 . (in Chinese with English abstract) [陈艳, 李宏庆, 刘敏, 陈小勇 (2010) 榕一传 粉榕小蜂间的专一性与协同进化. 生物多样性, 18 , 1-10.] 
Compton SG, Holton KC, Rashbrook VK, Van Noort S, Vincent SL (1991) Studies of Ceratosolen galili, a non-pollinating agaonid fig wasp. Biotropica, 23, 188-194.

Cook JM, Lopez-Vaamonde C (2001) Fig biology: Turning over new leaves. Trends in Ecology \& Evolution, 16, 11-13.

Cook JM, Rasplus J-Y (2003) Mutualists with attitude: Coevolving fig wasps and figs. Trends in Ecology \& Evolution, 18, 241-248.

Cook JM, West SA (2005) Figs and fig wasps. Current Biology, 15, R978-R980.

Cruaud A, Ronsted N, Chantarasuwan B, Chou LS, Clement WL, Couloux A, Cousins B, Genson G, Harrison RD, Hanson PE (2012) An extreme case of plant-insect co-diversification: Figs and fig-pollinating wasps. Systematic Biology, 61, 1029-1047.

Frankham R (1996) Relationship of genetic variation to population size in wildlife. Conservation Biology, 10, 1500-1508.

Galil J, Eisikowitch D (1968) On the pollination ecology of Ficus sycomorus in East Africa. Ecology, 49, 259-269.

Ganeshaiah KN, Kathuria P, Shaanker RU, Vasudeva R (1995) Evolution of style-length variability in figs and optimization of ovipositor length in their pollinatior wasps: A coevolutionary model. Journal of Genetics, 74, 25-39.

Ghana S, Suleman N, Compton SG (2017) Style length variation in male and female figs: Development, inheritance, and control of pollinator oviposition. Entomologia Experimentalis et Applicata, 162, 41-50.

Gu D, Compton SG, Peng YQ, Yang DR (2012) “Push” and "pull” responses by fig wasps to volatiles released by their host figs. Chemoecology, 22, 217-227.

Guan JM, Peng YQ, Yang DR (2007) Host sanctions in fig-fig wasp mutualism. Biodiversity Science, 15, 1569-1574. (in Chinese with English abstract) [管俊明, 彭艳琼, 杨大荣 (2007) 榕-蜂互惠关系中榕树对未传 粉榕小蜂的惩罚效应. 生物多样性, 15, 626-632.]

Haine ER, Martin J, Cook JM (2006) Deep mtDNA divergences indicate cryptic species in a fig-pollinating wasp. BMC Evolutionary Biology, 6, 83-94.

Herre EA (1985) Sex ratio adjustment in fig wasps. Science, 228, 896-898.

Herre EA (1987) Optimality, plasticity and selective regime in fig wasp sex ratios. Nature, 329, 627-629.

Herre EA, Jander KC, Machado CA (2008) Evolutionary ecology of figs and their associates: Recent progress and outstanding puzzles. Annual Review of Ecology, Evolution, and Systematics, 39, 439-458.

Herre EA, Knowlton N, Mueller UG, Rehner SA (1999) The evolution of mutualisms: Exploring the paths between conflict and cooperation. Trends in Ecology \& Evolution, 14, 49-53.

Hossaert-McKey M, Proffit M, Soler CCL, Chen C, Bessiere JM, Schatz B, Borges RM (2016) How to be a dioecious fig: Chemical mimicry between sexes matters only when both sexes flower synchronously. Scientific Reports, 6, 21236.

Hossaert-McKey M, Soler C, Schatz B, Proffit M (2010) Floral scents: Their roles in nursery pollination mutualisms. Chemoecology, 20, 75-88.

Huang JF, Xu R, Peng YQ (2018) Progress on the breakdown of one-to-one rule in symbiosis of figs and their pollinating wasps. Biodiversity Science, 26, 295-303. (in Chinese with English abstract) [黄建峰, 徐 睿, 彭艳琼 (2018) 榕-传粉榕小蜂非一对一共生关系 的研究进展. 生物多样性, 26, 295-303.]

Jander KC, Dafoe A, Herre EA (2016) Fitness reduction for uncooperative fig wasps through reduced offspring size: A third component of host sanctions. Ecology, 97, 2491-2500.

Jander KC, Herre EA (2010) Host sanctions and pollinator cheating in the fig tree-fig wasp mutualism. Proceedings of the Royal Society B: Biological Sciences, 277, 1481-1488.

Jander KC, Herre EA (2016) Host sanctions in Panamanian Ficus are likely based on selective resource allocation. American Journal of Botany, 103, 1753-1762.

Jander KC, Herre EA, Simms EL, Irwin R (2012) Precision of host sanctions in the fig tree-fig wasp mutualism: Consequences for uncooperative symbionts. Ecology Letters, 15, $136 z 1369$.

Jander KC, Steidinger BS (2017) Why mutualist partners vary in quality: Mutation-selection balance and incentives to cheat in the fig tree-fig wasp mutualism. Ecology Letters, 20, 922-932.

Janzen DH (1979) How to be a fig. Annual Review of Ecology and Systematics, 10, 13-51.

Kjellberg F, Jousselin E, Bronstein JL, Patel A, Yokoyama J, Rasplus JY (2001) Pollination mode in fig wasps: The predictive power of correlated traits. Proceedings of the Royal Society B: Biological Sciences, 268, 1113-1121.

Li ZT, Peng YQ, Wen XL, Jander KC (2016) Selective resource allocation may promote a sex ratio in pollinator fig wasps more beneficial for the host tree. Scientific Reports, 6, 35159.

Machado CA, Robbins N, Gilbert MTP, Herre EA (2005) Critical review of host specificity and its coevolutionary implications in the fig/fig-wasp mutualism. Proceedings of the National Academy of Sciences, USA, 102, 6558-6565.

Moe AM, Clement W, Weiblen GD (2012) Rapid evolution of pollinator-mediated plant reproductive isolation. In: Rapidly Evolving Genes and Genetic Systems (eds Singh RS, Xu J, Kulathinal RJ), pp. 266-273. Oxford University Press, Oxford.

Molbo D, Machado CA, Sevenster JG, Keller L, Herre EA (2003) Cryptic species of fig-pollinating wasps: Implications for the evolution of the fig-wasp mutualism, sex allocation, and precision of adaptation. Proceedings of the National Academy of Sciences, USA, 
100, 5867-5872.

Murray MG (1985) Figs (Ficus spp.) and fig wasps (Chalcidoidea, Agaonidae): Hypotheses for an ancient symbiosis. Biological Journal of the Linnean Society, 26, 69-81.

Peng YQ, Duan ZB, Yang DR, Rasplus JY (2008) Co-occurrence of two Eupristina species on Ficus altissima in Xishuangbanna, SW China. Symbiosis, 45, 9-14.

Proffit M, Schatz B, Bessiere JM, Chen C, Soler C, Hossaert- Mckey M (2008) Signalling receptivity: Comparison of the emission of volatile compounds by figs of Ficus hispida before, during and after the phase of receptivity to pollinators. Symbiosis, 45, 15-24.

Rubin BER, Moreau CS (2016) Comparative genomics reveals convergent rates of evolution in ant-plant mutualisms. Nature Communications, 7, 12679.

Sharp PM, Li WH (1989) On the rate of DNA sequence evolution in Drosophila. Journal of Molecular Evolution, 28, 398-402.

Smith JM (1982) Evolution and the theory of games. Cambridge University Press, Cambridge.

Soler CCL, Proffit M, Bessiere JM, Hossaert-McKey M, Schatz B (2012) Evidence for intersexual chemical mimicry in a dioecious plant. Ecology Letters, 15, 978-985.

Souto-Vilarós D, Machac A, Michalek J, Darwell CT, Segar ST (2019) Faster speciation of fig-wasps than their host figs leads to decoupled speciation dynamics: Snapshots across the speciation continuum. Molecular Ecology, 28, 3958-3976.

Thompson RCA (2013) Parasite zoonoses and wildlife: One health, spillover and human activity. International Journal for Parasitology, 43, 1079-1088.

Wang B, Geng XZ, Ma LB, Cook JM, Wang RW (2014) A trophic cascade induced by predatory ants in a fig-fig wasp mutualism. Journal of Animal Ecology, 83, 1149-1157.

Wang RW, Dunn DW, Sun BF (2014) Discriminative host sanctions in a fig-wasp mutualism. Ecology, 95, 1384 $-1393$.

Wang RW, Ridley J, Sun BF, Zheng Q, Dunn DW, Cook J, Shi L, Zhang YP, Yu DW (2009) Interference competition and high temperatures reduce the virulence of fig wasps and stabilize a fig-wasp mutualism. PLoS ONE, 4, e7802.

Wang RW, Sun BF, Yang Y (2015a) Discriminative host sanction together with relatedness promote the cooperation in fig-fig wasp mutualism. Journal of Animal Ecology, 84, 1133-1139.

Wang RW, Sun BF, Zheng Q, Shi L, Zhu LX (2011) Asymmetric interaction and indeterminate fitness correlation between cooperative partners in the fig-fig wasp mutualism. Journal of the Royal Society Interface, 8, 1487-1496.
Wang RW, Wen XL, Chen C, Shi L, Compton SG (2015b) Spatial heterogeneity and host repression in fig-fig wasp mutualism. Science China: Life Sciences, 58, 492-500.

Weiblen GD (2002) How to be a fig wasp. Annual Review of Entomology, 47, 299-330.

Weiblen GD (2004) Correlated evolution in fig pollination. Systematic Biology, 53, 128-139.

Xu R, Zhang Y, Peng YQ, Yang DR (2016) Reproductive characteristics of pollinator and cheater wasps that utilize the female flowers of Ficus altissima. Acta Ecologica Sinica, 36, 1134-1140. (in Chinese with English abstract) [徐睿，张媛，彭艳琼，杨大荣 (2016) 高榕雌花期传粉榕小蜂和欺骗性小蜂的繁殖特点. 生 态学报, 36, 1134-1140.]

Yang DR, Xu L, Peng YQ, Wei ZD, Duan ZB (2004) Species composition and diversity of fig wasps and figs in Yunnan. Biodiversity Science, 12, 611-617. (in Chinese with English abstract) [杨大荣, 徐䂞, 彭艳琼, 魏作东, 段柱标 (2004) 云南省榕小蜂和榕树的物种 组成及多样性. 生物多样性, 12, 611-617.]

Yang LY, Machado CA, Dang XD, Peng YQ, Yang DR, Zhang DY, Liao WJ (2015) The incidence and pattern of copollinator diversification in dioecious and monoecious figs. Evolution, 69, 294-304.

Yu DW, Ridley J, Jousselin E, Herre EA, Compton SG, Cook JM, Moore JC, Weiblen GD (2004) Oviposition strategies, host coercion and the stable exploitation of figs by wasps. Proceedings of the Royal Society B: Biological Sciences, 271, 1185-1195.

Zhang DY (2000) Researches on Theoretical Ecology. Higher Education Press, Beijing. (in Chinese with English abstract) [张大勇 (2000) 理论生态学研究. 高 等教育出版社, 北京.]

Zhang DY, Lin K, Hanski I (2004) Coexistence of cryptic species. Ecology Letters, 7, 165-169.

Zhang T, Miao BG, Wang B, Peng YQ, Darwell CT (2019) Non-pollinating cheater wasps benefit from seasonally poor performance of the mutualistic pollinating wasps at the northern limit of the range of Ficus microcarpa. Ecological Entomology, 044, 844-848.

Zhang XW, Dunn DW, Wen XL, Sun BF, Wang RW (2019) Differential deployment of sanctioning mechanisms by male and female host trees in a gynodioecious-fig wasp mutualism. Ecology, 100, e02597.

Zhang Y, Peng YQ, Yang DR (2014) Effects of foundress number, foundresses entry interval and non-pollinating wasps on clutch size and offspring sex ratio of pollinating fig wasps (Hymenoptera: Agaonidae). Acta Entomologica Sinica, 57, 587-593. (in Chinese with English abstract) [张媛, 彭艳琼, 杨大荣 (2014) 母 代雌蜂数、进果时间及非传粉小蜂对传粉榕小蜂后代 数量及性比的影响. 昆虫学报, 57, 587-593.]

(责任编委：张大勇 责任编辑：时意专) 